\title{
Gent féminine et pérennisation des valeurs traditionnelles dans les Monts Mandara: cas de la femme Podokwo et Guemjek à travers le mariage et les tâches quotidiennes
}

\author{
Tougbouné* \\ Université de Maroua, Cameroun
}

*Corresponding Author: Tougbouné, Université de Maroua, Cameroun

\begin{abstract}
In the Mandara Mounts, traditional societies are governed by norms and principles which differ in various points, especially the ones governing the so-called modern traditions. It is thus a place par excellence to express strong feeling for social and traditional values transmitted from generation to generation. At times, "less Knowledgeable" studies and excessive consideration which are sometimes derogatory tend to view the "Kirdi" woman as a victim of a merciless society. However, she is rather viewed in a two-fold way: she is not only a too little creature to her husband and subject to unlimited exploitation, but also and particularly the family adviser and keeper of traditional values. The sociofamily life is as such inspired by this bouncy of contiguity. In this respect, the Mounts Mandara woman is such an essential agent on that note. As time goes by, the Podokwo woman and the Guemjek woman have to make up for new reality trends of modernity so as to adapt to them.
\end{abstract}

Keywords: Podokwo, Guemjek, sustaincibility, traditional values, marriage, culture, society, Mounts Mandara, Northern Cameroon, Kirdi.

Résumé: Dans les Monts Mandara, les sociétés traditionnelles sont régies par des normes et principes qui diffèrent en beaucoup des points de ceux et celles qui régissent les sociétés dites modernes. C'est le lieu par excellence de l'attachement aux valeurs sociales et traditionnelles transmises de génération en génération. Des études parfois "nä̈ves » et des considérations souvent péjoratives et excessives ont tendance à faire de la femme "Kirdi » la victime d'une société impitoyable. Mais en réalité, elle se présente sous une double étiquette: elle est non seulement mineure soumise à l'homme et corvéable à souhait, mais aussi et surtout conseillère de la famille et gardienne des valeurs traditionnelles. La vie socio-familiale est conditionnée par cette dynamique de continuité et donc la femme des Monts Mandara est un agent essentiel. Prises par le cours du temps, la femme Podokwo et la femme Guemjek sont appelées à s'adapter aux nouvelles réalités de la modernité et de s'y approprier.

Mots-clés: Podokwo, Guemjek, pérennisation, valeurs traditionnelles, mariage, culture, société, Monts Mandara, Cameroun septentrional, Kirdi.

\section{INTRODUCTION}

Les groupes ethniques des MontsMandara, depuis la période coloniale (1902-1959) jusqu'à nos jours, n'ont jamais cessé d'intéresser les anthropologues, sociologues, linguistes et historiens. Différents instituts de recherches à l'instar d'Office de la Recherche Scientifique et Technique Outre-Mer (ORSTOM), l'Institut de Recherche pour le Développement (IRD) et bon nombre de Projets [1] ont soutenue des recherches dans cette zone. Les MontsMandara sont un ensemble de plateaux et massifs granitiques et volcaniques d'une altitude moyenne de $900 \mathrm{~m}$, situé entre $9^{\circ}$ et $10^{\circ}$ Nord, $13^{\circ}$ et $14^{\circ}$ de longitude Est [2]. La vie dans un tel milieu exige une adaptation d'autant plus difficile que l'exigüité de l'espace, les caractères accidentés du relief et de la pression démographique ne permettent guère l'épanouissement des activités économiques [3]. Les populations sont obligées de composer avec ce qu'offre la montagne pour leur survie. Les MontsMandara sont divisés en deux parties : la partie septentrionale qui couvre la subdivision de Mora et une partie de la subdivision de Mokolo et la partie méridionale couvrant, quant à elle, la subdivision de guider qui est à cheval entre la région de l'Extrême-Nord Cameroun et la région du Nord Cameroun actuelles. Dans cet article, nous nous intéressons uniquement à la partie septentrionale des Monts Mandara pour deux raisons. Premièrement, pour l'ancienneté de peuplement de cette partie, une ancienneté qui a permis aux 
Gent féminine et pérennisation des valeurs traditionnelles dans les Monts Mandara: cas de la femme podokwo et guemjek à travers le mariage et les tâches quotidiennes

occupants de mettre en place une civilisation séculaire et des sites refuges contre les attaques des étrangers. Et deuxièmement, pour la densité et la diversité de la population qui font la richesse et l'opportunité de la zone. Les Monts Mandara abritent une mosaïque de peuples : les Podokwo, les Mouktété, les Moura, les Vamé/Mbrémé, les Ouldémé, les Mada, les Zoulgo, lesMokyo, les Molko, les Mboko, les Matakam, les Guemjek et bien d'autres [4]. En raison de fractionnement aigu de ces peuples, certains auteurs ont préféré les regrouper sur la base de leurs histoires migratoires ou des similitudes que représentent leurs langues leurs rites et autres pratiques sociales [5]. Tous ces peuples sont désignés, àtort ou à raison, par le vocable ou patronyme "Kirdi ». Ainsi, le regard posé sur eux, souffre d'un prejudgeet d'une comparaison permanente avec les civilisations jugées plus brillantes développés en plaine [6]. Pourtant, ces peuples dits «Kirdi » se sont dotés, chacun en ce qui le concerne, d'une culture, d'une tradition tout court, et donc d'une civilisation qui font leurs fiertés. Pour montrer cette dynamique culturelle, nous nous sommes penché sur la gent féminine, à travers certains aspects de leurs traditions.

Des études souvent superficielles et hâtives ont fait de la femme «Kirdi » la victime d'une société impitoyable. Certes, dans les sociétés traditionnelles du Cameroun septentrional, ce qui d'emblée attire l'attention, c'est qu'il y'a un monde des femmes en marge de celui des hommes. La division du travail et la répartition de statuts laissent les tâches les plus ardues et audacieuses à la gent masculine et les travaux exigeant parfois de la patience et de la précision à la gent féminine. Toutefois, dans certaines ethnies, dans les MontsMandara, les femmes n'échappent guère aux travaux qui, normalement, réservés aux hommes. La société traditionnelle est régie par des lois, des normes et principes qui n'ont rien à voir avec ceux des sociétés dites modernes. Elle est le lieu par excellence de l'attachement aux valeurs sociétales léguées de génération en génération. La vie socio-familiale est conditionnée par une continuité dont la femme est un agent essentiel. La femme devient ipso-facto gardienne des valeurs traditionnelles de par ses innombrables tâches quotidiennes et saisonnières, son savoir-faire et son statut socio-familial. La femme traditionnelle, à cet effet, symbolise cette dynamique culturelle. Elle assure la permanence et la sauvegarde des valeurs traditionnelles contre des multiples et diverses mutations qu'impose le temps. L'objectif visé par cet article est de mettre en exergue les caractères permanents de certains aspects socioculturels dans les MontsMandara à travers le regard porté sur la femme Podokwo et la femme Guemjek.

\section{SOCIETEPODOKWO ET SOCIETEGUEMJEK DANS LES MONTSMANDARA}

Dans les MontsMandara, toutes les ethnies sont délimitées par les massifs, la langue, les us et coutumes, les techniques (arts et cosmogonie). Chaque ethnie est dotée d'une culture, des ethnonymes et des patronymes. Chaque ethnie représente une société spécifique. Notre choix est porté sur les sociétés podokwo et guemjek. Ces deux sociétés sont situées aux deux extrémités des Monts Mandara septentrionaux. Les Podokwo sont à l'Extrême-Nord des Monts Mandara (voisins directs de Mandara) et les Guemjek à l'Extrême-Sud des Monts Manadara (voisins directs des Moufou).

\subsection{La Société Podokwo et les Podokwo}

Les Podokwo sont divisés en plusieurs factions, à savoir les Makoulahé, les Kassa, les Namba, les Maja, les Gougna, les Tlala-Dabara, les Ouzlegaya, les Skwala et les Oudjila [7]. Chaque faction constitue une entité socio-politique lignagère. Les exigences sociales comme celles de la préservation des acquis ancestraux, la défense du territoire, la résolution des litiges selon les coutumes ancestrales, la consolidation des alliances, la protection de la population et la prévention des événements malheureux, (maladie, disette et bien d'autres encore) ont entrainé l'émergence des dignitaires traditionnels.L'existence du chef, communément appelé Tlewandala,n'est pas synonyme d'une autorité politique réelle. C'est un pouvoir politique flou et lâche [8]. Cependant, la société podokwo n'est pas une société anarchique. Elle est gérée de manière traditionnelle par les dignitaires dont les titres revêtent des valeurs socio-culturelles. Ces titres reflètent les fonctions qu'ils exercent dans la société. Tous ces dignitaires exercent des fonctions socio-religieuses. C'est la raison pour laquelle les Podokwo leur attachent une grande considération. Héréditaires par nature, ces titres sont attribués selon les règles et principes de la tradition podokwo. Cette grande estime et ce respect de l'héritage traduisent parfaitement les valeurs socio-culturelles des titres. Par exemple, les titres BatatipNamgaouSlalawa(faiseur de pluie), Batakud-kaya(desservant de l'esprit de la maison), Tlewandala-Mota (grand prêtre chef des cultes) et bien d'autres encore, ne font pas l'objet d'achat [9]. 
Gent féminine et pérennisation des valeurs traditionnelles dans les Monts Mandara: cas de la femme podokwo et guemjek à travers le mariage et les tâches quotidiennes

Ces titres sont les reflets de leur société et le poids de leur tradition. Les Podokwo ne désignent jamais des hommes ordinaires par ces titres. L'attribution des titres dans la société podokwofait l'objet des cérémonies rituelles au cours desquelles, les anciens du village rappellent à celui à qui est conféré le titre ces points importants: le code de comportement, les us et coutumes et les tâches qui lui incombent [10]. La place des dignitaires dans la société Podokwo précise le mieux la valeur socioculturelle de leurs titres. Les dignitaires traditionnels détiennent l'autorité morale, religieuse et socioculturelle. De par leurs titres, nous avons: ceux qui prédisent l'avenir et prient pour conjurer les forces redoutables, ceux qui guérissent le mal, ceux qui exécutent les rites et protègent le village. Chacun de ces dignitaires dispose d'un objet, symbole de son pouvoir et de sa puissance: les cailloux blanchâtres ou noirâtres, les cauris, les bâtonnets fétiches, les boucles de fer ou les crabes [11]. Le chef de clan est le seul habilité à accomplir les rites agraires. Il donne le signal de semailles et de récoltes. Cependant, le Tlewandala-Mota joue le rôle du grand prêtre, il est le gardien d'objets sacrés qui symbolisent l'union de tous les Podokwo. Prêtre du tombeau du héros fondateur ou tout au moins prêtre suprême du culte des ancêtres, il officie la fête triennale au cours de laquelle est sacrifié un taureau. En l'honneur donc des ancêtres, le grand prêtre immole un taureau auprès du tombeau du fondateur du clan. Les quatre sabots et une partie de la peau de l'animal sont enfouis dans le tombeau et les cornes sont plantées sur ledit tombeau. Le grand prêtre fait enfin le tour de tous les grands rochers qui dominent le pays podokwo et y dépose de la terre pétrie. Ce rite est suivi des libations d'eau de la farine du mil rouge, signe de prospérité et de protection du clan [12]. Ce culte exécuté par ce dignitaire est d'une signification profonde et d'une grande portée. Selon les Podokwo, le taureau sacrifié en l'honneur des ancêtres représente une offrande des vivants aux aïeux afin d'assurer la bonne santé, la paix dans les massifs, la prospérité et la procréation [13].

Les rites agraires sont également un domaine exclusif des dignitaires. Ils se passent une fois l'an. Le chef de chaque clan, à cette occasion, est convié à offrir un taureau, les repas et le vin traditionnel pour la fertilité de la terre. Chez les Podokwo, personne ne peut semer ou récolter sans le signal du chef des cultes agraires. Cet attachement des Podokwo aux valeurs traditionnelles explique l'importance qu'ils accordent à leurs dignitaires. Dans la pharmacopée traditionnelle, le pouvoir de prédire l'avenir par divination et d'exécuter les rites n'appartient pas aux hommes ordinaires [14]. Toutes ces considérations concourent à placer les dignitaires traditionnels au premier rang et à faire d'eux la poutre de la société traditionnelle. Les respects, les prestiges, et le dévouement que les Podokwo réservent à leurs dignitaires ne sont pas de l'arrière main. Ils attestent leur véritable place au sein de la société. Ce qu'il importe de retenir, c'est que la société Podokwo a bâti ses propres règles et principes quant à l'attribution des titres des dignitaires qu'à leurs rapports avec la population.

\subsection{La société guemjek et les Guemjek}

La société guemjek est divisée en deux classes, à savoir le Massakamburoou la basse classe et le Gwala bay ou MalaGma, la classe détentrice du pouvoir [15]. C'est de cette dernière classe dont ressortent tous les titres dans la société guemjek. Le chef porte le titre de Bay. Il est aussi désigné par certains expressions, Ngalaka(Majesté), Féték, Kerngai(étenel), Hotokom(sommet)[16]. Ici, dans cette société, le chef n'est qu'un Primus inter pares,c'est-à-dire le premier entre les égaux. Il n'a que l'autorité morale et religieuse. L'autorité hiérarchique est confiée à un clan étranger nouvellement arrivé sur le massif. Selon les Guemjek, en confiant l'autorité au clan étranger, cela faciliterait leur soumission et ils se mettront aux services des « autochtones » [17]. Pour les Guemjek, se mettre au service des esprits de la montagne et des puissances invisibles est une tâche très délicate et comporte beaucoup d'interdits à observer [18]. Dans la société guemjek, il existe environ sept types de titulatures: le Bay Gma[19] (chef de massif), le MalaYam[20], le Mbulum[21] (le desservant de l'esprit de lieu), le Malalawadara[22] (le desservant de l'esprit de l'ancêtre du lignage), le Mahram[23] (le divin guérisseur), le Kleng[24] (le chef-voyant), leKerldagam[25] (le desservant du dieu de la porte).La valeur socio-culturelle des titres des dignitaires chez les Guemjek est tributaire du code de leur comportement dans la société. Toutefois, la puissance et le pouvoir que le dignitaire dispose font également de lui un homme craint et respectueux.Il est facile de distinguer les dignitaires des hommes ordinaires, de par leurs symboles de pouvoir et de puissance. Ces symboles sont entre autres: une toge rouge, un long bonnet rouge, des colliers en cuir et en dent de panthère, un sabre d'un arbre fétiche, pour ne citer que ceux-là. Les dignitaires traditionnels, bien qu'ils ne détiennent pas totalement le pouvoir politique, ils sont tout de même présents dans certains domaines chers à la 
Gent féminine et pérennisation des valeurs traditionnelles dans les Monts Mandara: cas de la femme podokwo et guemjek à travers le mariage et les tâches quotidiennes

société. Ils détiennent l'autorité morale, religieuse et socio-culturelle. De par leurs titres et leurs tâches, les dignitaires occupent le premier rang. Les Guemjek, vu l'attachement mordicus qu'ils affichent vis-à-vis de leur tradition, ne peuvent reléguer leurs dignitaires au second plan. Les dignitaires sont sollicités à tout moment et en toute saison. Le calendrier des semailles et des récoltes, la date des fêtes, les cérémonies rituelles sont l'œuvre des dignitaires [27]. Pour donner plus d'estimes aux dignitaires, les Guemjek ont choisis d'élever le domicile de leur Bay sur le plus haut sommet, et au- dessus des habitats du reste de la population [28]. Ce choix n'est pas le fait du hasard. Chez les Guemjek, la symbolique du « haut lieu » et du «bas lieu » est pleine de significations. Le « haut lieu» ou le sommet est selon eux un lieu sacré où résident les esprits et les puissances surnaturelles. C'est un lieu par excellence de possibilité de communiquer avec les esprits. Il traduit, par ce fait, le pouvoir, la masculinité, la pureté, la prééminence, la force, la supériorité et la primauté [29]. Ceci justifie le choix du domicile de leurs dignitaires qui se trouve toujours sur le plus haut sommet. Malgré l'immensité des tâches et des interdits [30] qui incombe aux dignitaires, ils ne bénéficient d'aucune rémunération. Ils reçoivent tout de même la main d'œuvre gratuite et volontaire, des dons et des cadeaux. Ils bénéficient cependant, de l'honneur, du respect de toutes les populations guemjek et leurs familles profitent d'un certains prestiges et d'une certaine immunité. C'est dans ces sociétés avec ces dignitaires que la femme Podokwo et la femme Guemjek vont jouer leurs rôles.

\section{Femme Podokwo et Femme Guemjek et LA Sauvegarde des Valeurs TRADITIONNELLES}

Dans la société patriarcale où l'homme établissait l'éthique de la société à partir du sexe, la femme se présentait sous une double facette. Autant elle est considérée comme personne mineure, soumise et utile à sa sensibilité à l'homme, autant avec sa sagesse, son expérience naturelle sa sensibilité et son ingéniosité, elle pourrait s'imposer dans la famille et devenir la conseillère, le pivot de la famille et la gardienne de la tradition. Nous l'observerons à travers la femme Podokwo et la femme Guemjek traditionnelles.

\subsection{La femme Podokwo à travers le mariage et le statut socio-familial: garante de la Iradition}

Les Podokwo attachent une grande importance à la femme. Le sérieux de leur société réside dans la solidarité des alliances matrimoniales. Le choix d'une fiancée intéresse tous les membres d'une famille. Les Podokwo établissent un parallèle de l'identité entre la fertilité de la terre et la fécondité de la femme. C'est la raison pour laquelle, les épouses et les enfants constituent leurs véritables richesses et la terre leur ultime attachement.Le mariage communément appelé Moundwaou Gui-Nissa, n'est pas une simple occasion de jouissance, ni une simple alliance entre deux personnes, mais il constitue l'un des véritables aspects de la culture. Il permet l'union de deux familles, et parfois de deux clans, deux ethnies voire deux sociétés. C'est pourquoi, les Podokwo ne se lancent pas aveuglement dans l'aventure conjugale. Ils passent toujours par le canal de la divination pour connaître tous les contours (fécondité, prospérité, éducation et chance) de la jeune fille et par le canal des enquêtes sérieuses sur la famille de la jeune fille afin de savoir sur elle [31].La durée des fiançailles, généralement, comprise entre trois et cinq ans, témoigne bien leur ferme attachement au mariage et à la vie conjugale. Pour les Podokwo, la femme est la lumière de la famille, et la famille, celle de la société [32]. La famille est dite famille que lorsqu'il y a la présence d'une femme ou lorsque la femme a laissé ses traces, des progénitures.La femme Podokwo est sujette à plusieurs sortes de mariages: le mariage par consentement des amants après une longue période des fiançailles, le mariage par héritage ou par principes coutumiers après le veuvage et le mariage par rapt.

Le mariage par consentement des amants fait appel à une longue période d'observation et d'étude mutuelles. La jeune fille ou Doua à marier a la forme ronde et solide, et doit en moyenne avoir seize à dix-huit ans. Les funérailles, les marchés et les lieux bucoliques constituent les véritables occasions de rencontre entre filles et garçons. Durant toute la période des fiançailles, le jeune garçon ou Douhoulaest permanemment au service de sa belle-famille [33]. C'est la fille qui décide de ratifier le choix fait au début des fiançailles. Toutefois, la jeune fille doit garder et préserver sa chasteté pour pouvoir rentrer plus tard en possession de la dot. Il peut cependant arriver que la fille change de décision et s'amourache d'une autre personne, cela n'entrave en rien les exigences que nous avons précédemment évoquées. Comme l'exige la tradition podokwo, la femme ou Nissa, avant d'obtenir le mariage, doit nécessairement passer au crible de fiançailles avec tous les rites y afférents. C'est 
Gent féminine et pérennisation des valeurs traditionnelles dans les Monts Mandara: cas de la femme podokwo et guemjek à travers le mariage et les tâches quotidiennes

pourquoi le rapt est réprimandé.En revanche, le mariage par héritage ou par exigence de la tradition, se passe après la mort du mari plus précisément pendant le veuvage [34]. La veuve, à cet effet, choisit elle-même un membre de la famille de son défunt mari. Par simple geste qui consiste à donner de l'eau à la farine du mil rouge à l'un des membres de la belle-famille, et le mariage est conclus à l'immédiat. Ce rite se déroule en présence des anciens, des sages et les membres des deux familles concernées.Le mariage par rapt, cependant, existe dans la société Podokwo, mais il n'est pas fréquent du fait qu'il soit toujours réprimandé. Ce type de mariage est source de beaucoup de conflits: le conflit MakoulahéKassa en 1924 d'une part et le conflit Namba-Makoulahé en 1939 [35] d'autre part en sont des exemples marquants.

Pour tous les mariages ainsi évoqués, c'est la dot qui confirme leur légalité. Chez les Podokwo, comme le veut la tradition, pour doter une fille, un bœuf et deux chèvres sont impérativement exigés [36]. Le choix porté sur ces deux espèces de bête est significatif. Pour eux, le bœuf est un animal sacré qui permet d'être en contact avec les ancêtres et la chèvre symbolise la fécondité. La femme dotée de la sorte pourrait bénéficier de la protection permanente des ancêtres et avoir un ventre fécond [37]. Ce choix du bœuf dans les sacrifices est fait par le grand prêtre podokwo.

La durée de jouissance du mariage varie d'un clan à un autre. La plus longue durée est d'un mois et toujours sanctionnée par une nuit de noce. Le mariage en pays Podokwo, avec toutes ses spécificités et tous ses rites, est ce par quoi la jeune fille devient épouse, ménagère, mère et maîtresse de maison. Tout ceci constitue son statut socio-familial. Toutefois, la structure du foyer (polygamique ou monogamique) joue un grand rôle sur ledit statut. Dans le cas de la polygamie, son statut est fonction de la place qu'elle occupe selon l'ordre de son arrivée au foyer. La Tlatawaou la première femme du foyer, est en même temps mère, conseillère et pédagogue des valeurs traditionnelles socio- familiale [38]. Elle reste le modèle pour les autres épouses. Tous ses gestes et faitsdoivent minutieusement être suivis et imités par les autres coépouses [39]. Cependant, celles-ci ne sont pas laissées pour compte, même si la Tlatawaest celle par qui dépendent la stabilité de la famille, la bonne éducation des jeunes filles qui résident dans l'art culinaire, les mœurs et la morale de la société podokwo et tout ce qui est lié à la culture et enfin la sauvegarde de tout ce qui est valeur familiale.

Les rites liés à la grossesse, à l'accouchement, au traitement du nouveau-né et l'attribution du nom, témoignent, à plusieurs considérations, le vif du statut socio-familial de la femme Podokwo. Cependant, le veuvage qui est une période intermédiaire entre le décès de l'époux et le moment du remariage, n'est pas exclu du statut de la femme dans la famille. La veuve ne doit pas quitter la famille de son défunt mari. Elle est contrainte d'épouser, si possible le jour même du deuil, l'un des membres de la famille de son défunt époux.De la période des fiançailles jusqu'au veuvage, la femme Podokwo est d'une part l'objet et d'autre part sujet des coutumes et traditions de sa société. De tout ce qui précède, la femme Podokwo reste et demeure le noyau dur de la tradition. Elle reste cet être par qui, certains aspects de la tradition se pérennisent et se sauvegardent.

Au même titre que d'autres femmes montagnardes, la femme Podokwo sacrifie son temps aussi aux travaux domestiques qu'aux travaux champêtres. Il est difficile d'évaluer les tâches de la femme Podokwo en termes de statistiques, car presque la totalité des travaux et préoccupations familiaux lui revient (Tableau ${ }^{\circ} 1$ ). A la maison, la femme Podokwo, en plus du travail culinaire, avec tout ce que cela comporte, est appelée à apporter aux enfants, soin et attention afin de les élever à la dure et de leur inculquer une éducation à la tradition Podokwo. La jeune fille est entièrement sous la responsabilité de sa mère. La mère surveille et contrôle les faits et gestes de sa fille afin de ne point tomber sous le coup des coutumes. Lachasteté doit être préservée jusqu'au mariage. Une jeune fille qui a perdu sa chasteté non seulement elle est réprimandée sévèrement, mais aussi elle ne bénéficiera plus de la dot [40]. La femme Podokwo apprend à sa jeune fille tout ce qui est valeur traditionnelle socio-familiale. Elle devient par ce fait même nourricière et pédagogue. Les saisons et les circonstances ne lui laissent aucun répit.

Dans la société Podokwo, la production agricole vise particulièrement à satisfaire les besoins alimentaires familiaux et les besoins socio-culturels et religieux. Les denrées alimentaires obtenues sont utilisées pour la consommation, lors des événements tels que les mariages, les décès, les naissances, les fêtes traditionnelles et les cérémonies sacrificielles [41]. Excepté le mil dont la conservation est assurée par l'homme, toutes les autres variétés de plantes sont gérées par les femmes 
Gent féminine et pérennisation des valeurs traditionnelles dans les Monts Mandara: cas de la femme podokwo et guemjek à travers le mariage et les tâches quotidiennes

et conservées dans leurs propres silos [42]. De toutes les plantes dont les travaux sont exclusivement réservés à la femme Podokwo, le tabac et le haricot attirent le plus notre attention. Ces deux plantes interviennent dans toute la vie socio-culturelle et religieuse des Podokwo. Le haricot intervient dans le confectionnent rituel de certains mets spéciaux et le tabac est le symbole d'alliance et de la paix [43]. C'est la raison pour laquelle, après la dot, les Podokwo exigent le mélange rituel de tabac qui témoigne l'alliance entre les deux familles [44]. L'économie de subsistance est de coutume et basée essentiellement sur l'agriculture. Les rapports de production qui existent entre l'homme et la femme dans cette agriculture sont complémentaires, mais avec prédominance de la femme. La femme Podokwo y consacre plus de temps que l'homme [45]. Selon les Podokwo, même pendant l'époque où l'agriculture n'existait pas encore, c'est la femme qui cueillait et déterrait les produits de la brousse [46]. Peu à peu, comme disait Odile Chatap: « la femme s'est mise à faire pousser les produits sans plus attendre que la nature voulût bien les offrir spontanément»[47]. Les Podokwo ont donc compris cette logique pour laisser tout naturellement les travaux champêtres à la femme. Symbole de la fécondité, elle a la puissance de donner vie et une main bénite pour travailler les champs. Ces qualités reproductrices et ces facultés de production déterminent la place de la femme Podokwo aux yeux de son mari et de la société.Froelich n'est pas loin de cette réalité lorsqu'il souligne: «Au pays Podokwo, leurs systèmes économiques ne permet pas l'accumulation des richesses sauf sous forme d'épouses et d'enfants » [48]. Cette place prépondérante de la femme et surtout ses nobles tâches sont encore plus déterminantes parce qu'elles permettent non seulement la survie et l'immortalité de la famille, mais aussi assurent la pérennité et la continuité des valeurs traditionnelles.

Néanmoins, ni les tâches quotidiennes et saisonnières, ni le savoir-faire de la femme Podokwo, ni le paysage du pays Podokwo qui ont attiré des visiteurs,mais ce sont tout simplement les valeurs esthétiques corporelles et les parures de la femme Podokwodans les danses traditionnelles, d'une part et d'autre part la chefferie traditionnelle d'Oudjila [49]. Evidemment, l'esthétique qui est l'ensemble des atours destinés au rehaussement de la silhouette féminine, à des fins de séduction, de satisfaction personnelle et d'obéissance aux exigences socio-culturelles, n'ont laissé pour compte la femme Podokwo. Les parures chez les Podokwo occupent une place prépondérante. Elles sont fruit des traitements de graminées. Le fil de coton, les franges de lianes, les torsades végétales et bien d'autres encore, constituent des effets décoratifs. À ceux-ci, s'ajoutent les colliers plats, les brins spiralés qui servent d'ornements au poignet (Mtsda), au-dessus du coude et aux aisselles (Bida), au cou (Taya), aux reins (Patcha), au-dessous des genoux, aux chevilles et aux oreilles[50]. Les parures constituent un véritable indicateur et signe de distinction socio-culturelle et de démarcation ethnique. Les divers ornements utilisés par la femme Podokwo, ainsi que le temps qu'elle accorde aux parures, traduisent son grand degré d'attachement à la tradition. La répétition circonstancielle ou événementielle de se parer traditionnellement, permet de sauvegarder et de pérenniser laculture.

Tableau1: Activités journalières et saisonnières de la femme Podokwo traditionnelle

\begin{tabular}{|c|c|c|}
\hline Journée ordinaire & Saison sèche & Saison des pluies \\
\hline $\begin{array}{ll}\text { - } & \text { Chercher de l'eau de grand } \\
\text { matin } \\
\text { - } & \text { Préparer le repas (matin et } \\
\text { soir) } \\
\text { - } \quad \text { Chercher les termites } \\
\text { - } \quad \text { Chercher du boisCauserie } \\
\text { éducative avec les enfants }\end{array}$ & $\begin{array}{ll}\text { - } & \text { Préparer le vin des récoltes } \\
\text { - } & \text { Sécher l'oseille de guinée, le } \\
& \text { sésame et le tabac } \\
\text { - } & \text { Récolter le mil et autres } \\
\text { - } & \text { Décortiquer les arachides } \\
\text { - } & \text { Confectionner les silos } \\
& \text { personnelsMettre les produits } \\
\text { dans les silos }\end{array}$ & $\begin{array}{ll}\text { - } & \text { Préparer le sol } \\
\text { - } & \text { Préparer le vin des semailles } \\
\text { - } & \text { Semer les produits } \\
\text { agricolesLabourer le champ } \\
\text { familial et le champ personnel }\end{array}$ \\
\hline
\end{tabular}

Sources: Ce tableau est conçu par nous-même à partir d'informations recueillies sur le terrain

\subsection{La femme Guemjek à travers le mariage et le statut socio-familial: garante de la tradition}

Chez les Guemjek, le mariage est fondamental. Il engage non seulement deux individus, mais aussi deux groupes de parentés unis par des échanges de biens et de services. Il favorise une certaine homogénéité culturelle et étend une zone de confiance et d'harmonie du fait du lien de sang qu'il scelle. La femme Guemjek est affectée par cinq types de mariages : le mariage par proposition des parents [51], le mariage par rapt [52], le mariage par la « marque» [53], le mariage par héritage [54]et le mariage par consentement des amants [55]. Dans la société Guemjek comme dans plusieurs sociétés montagnardes, le mariage est toujours accompagné d'un certain nombre de rites. Et c'est la 
Gent féminine et pérennisation des valeurs traditionnelles dans les Monts Mandara: cas de la femme podokwo et guemjek à travers le mariage et les tâches quotidiennes

femme qui détient le pouvoir dans ce domaine. Tous les gestes et symboles durant le mariage sont significatifs chez les Guemjek. Dans les rites relatifs au mariage, les vielles femmes sont prioritaires. Le choix d'une vielle femme pour présider les rites répond à plusieurs critères et conditions. La vielle femme doit d'abord être saine, c'est-à-dire qu'elle n'a pas transgressé les principes coutumiers et elle n'a jamais divorcé [56]. Toutes ces conditions concourent à faire du mariage, un événement bénit, accepté par la tradition et les esprits bienveillants. Elles concourent également à faire d'une vie conjugale, une période de longévité, destabilité et d'harmonie. La vielle femme ayant rempli ces conditions devient un symbole. Loin d'être un simple événement, le mariage est toute une civilisation, une tradition. La femme Guemjek, trouve alors satisfaction dans ce domaine qui, non seulement l'affecte directement, mais dont elle détient aussi le secret. Nous ne pourrons présentement détailler tous les rites relatifs au mariage chez les Guemjek. Cependant, ce qu'il convient de retenir c'est que, la femme Guemjek est au centre et que c'est elle qui garde le schéma traditionnel du mariage. Ce qui attire le plus l'attention dans tous les rites relatifs au mariage, c'est cette prédominance des symboles. Ce qui affecte la dote est également de nature symbolique. Nous avons par exemple pour la dot : les caprins, le tabac, la houe, du natron, des peaux, des tissus de coton filés traditionnellement, pour ne citer que ceux-là.

Avant d'acquérir son statut de femme de maison, la jeune mariée doit passer par un certain rite appelé rite de la transformation de la mariée en une femme. Ce rite est toujours officié par les femmes. Il a lieu trois mois après le mariage. Àcette circonstance, ce sont les femmes dont les premiers nés sont encore vivants qui officient [57]. Elles vont, à cet effet, mâcher des bandes d'écorces spécifiques. Et au cours de cette opération, il est préparé le couscous à la sauce du haricot. Les femmes désignées pour cette circonstance enlèvent à la mariée son cache-sexe de jeune fille pour lui attacher au tour des reins, des dizaines de rangées de cordes en peau de bœuf, et une bande d'écorce couvrant totalement son sexe. La jeune mariée est, par ce rite, devenue femme. Elle acquiert par ce fait même son statut d'épouse et de femme de maison. Elle est appelée à assumer certaines responsabilités liées à tout ce qui est valeur familiale. Avec la naissance d'un enfant, elle acquiert le statut de mère et maîtresse de maison. Elle est dès lors tenue de se conformer et de se soumettre aux us et coutumes, non seulement ceux qui sont relatifs à sa société, mais également ceux qui regardent sa nouvelle famille. Elle devient ipso-facto gardienne des valeurs traditionnelles par son statut et les tâches qui désormais l'attendent.

L'endogamie étant prohibée chez les Guemjek, les femmes choisies dans diverses sociétés forment un foyer polygamique hétérogène. Non seulement elles ont du mal à s'adapter entre elles, mais aussi à adopter les us et coutumes de la nouvelle famille. La jeune mariée, nouvellement arrivée dans la famille guemjek est mise à la disposition de sa belle-mère ou d'une femme dans sa belle-famille, pour mieux être façonnée selon les normes et principes guemjek [58]. Ainsi, les tâches quotidiennes et saisonnières (Tableau $\mathrm{n}^{0} 2$ ) de la femme guemjek, son savoir-faire, ses valeurs esthétiques corporelles et ses parures sont foncièrement liés aux us et coutumes.La femme Guemjek, en plus de ses préoccupations domestiques y compris l'éducation des enfants, est appelée à confectionner des objets d'art pour le besoin socio-familial. Cependant, elle s'intéresse moins aux objets de luxe soigneusement travaillés qu'aux productions destinées aux usages quotidiens et événementiels. C'est à travers ces productions que l'on peut apprécier le mieux l'ingéniosité de la femme Guemjek et son apport dans lasauvegarde des coutumes et traditions. La femme Guemjek s'illustre tant dans la poterie que dans la vannerie.La poterie, véritable industrie et art de la femme, nécessite une technique adéquate et une grande finesse. Par industrie, il faut ici comprendre l'ensemble des techniques ou procédés de fabrication: mouillage [59], pillage [60], modelage [61], façonnage [62] et cuisson [63]. Et par art, l'ensemble des canons esthétiques: le type [64], la forme et la décoration. La potière Guemjek produit à la fois des pots à usage religieux communément appelés Duwaet des pots à usage domestique.

Jadis, la potière façonnait les pots, juste pour le besoin de sa famille. Lorsque la production était devenue impressionnante elle la passait à la vente ou à l'échange contre le mil et bien d'autres produits alimentaire. Àbien des considérations, la poterie sert à des représentations des divinités destinées au culte et à la symbolisation d'autels des esprits tels que le Mbulumet le Lawdara. Certains récipients en terre cuite servent à faire des offrandes aux dieux et esprits du massif afin de solliciter une protection ou uneguérison. Les petits vases ont généralement le rôle purificateur. Ils sont fréquemment utilisés dans les sacrifices rituels. Outre le domaine religieux, la poterie intervient aussi dans le domaine culturel. Certains instruments de musiques et des danses traditionnelles, comme les flûtes, sont faits en terre cuite. La poterie est foncièrement ancrée dans la vie économique et socio-culturelle des 
Gent féminine et pérennisation des valeurs traditionnelles dans les Monts Mandara: cas de la femme podokwo et guemjek à travers le mariage et les tâches quotidiennes

Guemjek et est secondée par la vannerie. Elle consiste en la confection d'objets tissés en raphia, osier, rotin et bien d'autres encore. La vannière guemjek produit, à cet effet, des paniers divers et variés destinés aux travaux domestiques et champêtres, des couvercles à dimension moyenne pour fermer les récipients de repas et des couvercles à grande dimension pour fermer les grands vases.Au demeurant, par ses tâches quotidiennes et son savoir-faire, la femme Guemjek contribue d'une manière remarquable à la pérennité et à la continuité de certains aspects culturels de sa société.

Tableau2: Activités journalières et saisonnières de la femme Guemjek traditionnelle

\begin{tabular}{|c|c|c|}
\hline Journée ordinaire & Saison sèche & Saison des pluies \\
\hline $\begin{array}{ll}\text { - } & \text { Chercher de l'eau de grand } \\
\text { matin } \\
\text { - } & \text { Préparer le repas (matin et } \\
\text { soir) } \\
\text { - } \quad \text { Chercher les termites } \\
\text { - } & \text { Chercher du boisCauserie } \\
& \text { éducative avec les enfants }\end{array}$ & $\begin{array}{ll}\text { - } & \text { Préparer le vin des récoltes } \\
\text { - } & \text { Récolter le mil, le haricot et les } \\
\text { arachides } \\
\text { - } \\
\text { Décortiquer les arachides et le } \\
\text { haricot }\end{array}$ & $\begin{array}{llll}\text { - } & \text { Semer les } & & \text { produits } \\
\text { agricoles } & & \\
\text { - Aider souvent le mari à } \\
\text { labourer }\end{array}$ \\
\hline
\end{tabular}

Sources: Ce tableau est conçu par nous-même à partir des informations recueillies sur le terrain.

\section{CONCLUSION}

Dans cet article, il importait pour nous de nous plonger dans ce méandre géographique qu'est la partie septentrionale des Monts Mandara. L'accent est mis sur les questions relatives à la femme Podokwo et Guemjekdans leurs sociétés respectives. La physionomie de la femme Podokwo comme celle de la femme Guemjek telle qu'elle se dégage de notre analyse, à travers le mariage et les tâches quotidiennes, est celle des femmes restées très attachées àleurs modes de vie traditionnels. Les différentes sociétés qui ont fait l'objet de cette analyse ont représenté la femme sous divers visages. Les tâches quotidiennes et saisonnières de la femme Podokwo et Guemjek sont tributaires de la structure de leurs foyers respectifs. Selon les normes et principes de leurs sociétés et traditions, les rites jalonnent toute la vie et les activités de la femme traditionnelle. Le statut socio-familial de la femme Podokwo et de la femme Guemjek traditionnelles constitue un véritable stade où se jouent leurs contributions à la pérennisation des valeurs traditionnelles. Cet article ne saurait être un procès dirigé contre les dénigreurs des peuples «Kirdi » et de leur tradition, encore moins un éloge de la femme Podokwo et Guemjek. Le seul souci était de tenter une analyse socio-historique, à partir d'informations que nous avons pu glaner en évitant toute attitude de subjectivité et de passivité.

\section{REFERENCES}

[1] Mission pour le Développement Intégral des Monts Mandara (MIDIMA) ; Mission Méga Tchad, Projet Nord-Est Bénoué et bien d'autres.

[2] Louléo, J., «Emigration des Kirdi des Monts Mandara: le cas des Mafa de soulédé », Thèse de Doctorat de géographie, Université de Yaoundé I, 1994, p.15. Lire également à ce sujet Hallaire, A., Paysans Montagnards du Nord-Cameroun, les Monts Mandara, Paris, ORSTOM ; Halloire, A, Les Monts du Mandara au Nord de Mokolo et la plaine de Mora : étude géographique régionale, Yaoundé, ORSTOM, IRCAM.

[3] GiglaGarakcheme, «La résistance des Kirdi à l'autorité coloniale dans les Monts Mandara (NordCameroun): Fondements et modalités, 1902-1960 » Thèse pour le Doctorat/Ph.D. d'Histoire, Université de Maroua, 2013-2014, p.17.

[4] Boutrais, J., La colonisation des plaines par les Montagnards au Nord du Cameroun(Monts Mandara), Paris, ORSTOM, 1973, p.13. Pourplus d'informations à ce sujet, lire Boutrais, J., Le Nord du Cameroun : des Hommes une région, Paris, ORSTOM, Collection Mémoire n ${ }^{\circ} 102,1984$; Bontrais, J., Le Nord du Cameroun: bilan de dix ans de recherches, Yaoundé, ORSTOM, 1971 et Boulet, J. et al.Le Nord du Cameroun: bilan de dix ans de recherche, Vol.1, ONAREST/ISH

[5] GiglaGarakcheme, «La résistance des Kirdi à l'autorité coloniale dans les Monts Mandara (NordCameroun) : Fondements et modalités, 1902-1960» Thèse pour le Doctorat/Ph.D. d'Histoire, Université de Maroua, 2013-2014, p.19

[6] GiglaGarakcheme, "La résistance des Kirdi à l'autorité coloniale dans les Monts Mandara (NordCameroun): Fondements et modalités, 1902-1960 » Thèse pour le Doctorat/Ph.D. d'Histoire, Université de Maroua, 2013-2014, p.19

[7] Hoché Doulouva, «Migrations des populations dites Kirdi du Haut Mandara, des origines à nos jours », 
Gent féminine et pérennisation des valeurs traditionnelles dans les Monts Mandara: cas de la femme podokwo et guemjek à travers le mariage et les tâches quotidiennes

Thèse de Doctorat/Ph.D d'histoire non soutenue, Université de Yaoundé I, 2010-2011, p.19.

[8] Hoché Doulouva, «Migrations des populations dites Kirdi du Haut Mandara, des origines à nos jours », Thèse de Doctorat/Ph.D, Université de Yaoundé I, 2010-2011, p.19.

[9] Entretien avec Bastara Fidel, 65 ans, ancien secrétaire d'état civil, à Godigong le 11/10/2018

[10] Entretien avec DougdjéMarva, 70 ans, Tradi-praticien, à Kassa 1, le 10/09/2017

[11] Entretien avec MaviaWassa, 50 ans, Tradi-praticien à Kassa 1, le 10/09/2017

[12] Entretien avec MaviaWassa, 50 ans, Tradi-praticien à Kassa 1, le 10/09/2017

[13] Entretien avec DougdjéMarva, 70 ans, Tradi-praticien à Kassa 1, le 10/09/2017

[14] Pour plus d'informations à ce sujet, voir Mouchet, M., « Vocabulaires comparatifs de sept parlers du Nord- Cameroun » Bulletin de la société d'Etudes Camerounaises (BUSEC), 1953, n41-42, Mouchet, M., «Prospection ethnologique sommaire de quelques massifs de Mandara : massif de Guemjek » BUSEC, $1957, n^{\circ} 55$.

[15] Manassé, J., « Monographie historique d'un peuple du Mont Mandara : les Guemjek, des origines à nos jours », Mémoire de DIPES II d'Histoire, ENS de Yaoundé I, 2002-2003, pp.28-29.

[16] Entretien avec Kwisseng, 65 ans, chef de massif, à Shrem, le $1^{\text {er }} / 10 / 2017$

[17] Entretien avec Kwisseng, 65 ans, chef de massif, à Shrem, le $1^{\mathrm{er}} / 10 / 2017$

[18] Entretien avec Kwisseng, 65 ans, chef de massif, à Shrem, le $1^{\text {er }} / 10 / 2017$

[19] Bay Gmaou (chef de massif) est nommé à vie. Sa désignation obéit à plusieurs critères relatifs aux coutumes Guemjek. Ses attributions sont beaucoup plus religieuses que politiques. Il est desservant de l'autel du grand esprit de la montagne (Zkal). Il donne l'ordre de semailles et de récoltes. Il fixe le calendrier des fêtes et cérémonies rituelles. Il veille au respect des coutumes et traditions. Informations recueillies auprès de Kwisseng chef de massif, à Shrem le $1^{\mathrm{er}}$ octobre 2017.

[20] Mala Yam: Chef des pluies, il est celui qui dispose du pouvoir de faire tomber la pluie. Il se sert des pierres réfléchissantes «Kur Yam» il en existe trois types : Kur Yam ou pierre de pluie de sécheresse et KurHembedou pierre de tornade. Il est capable de provoquer la pluie même en saison sèche. Sous la pluie, il ne se mouille Jamais. Il a le pouvoir d'envoyer la foudre là où il veut. Informations recueillies auprès de Mapjak, faiseur de pluie, à Guguleng le 07/10/2017.

[21] Mbulumou desservant de l'esprit de lieu. Il est l'esprit de lieu d'un lignage que l'ancêtre fondateur a domestiqué. Il se manifeste à travers des tourbillons et des grondements sonores. Il a pour rôle de protéger les membres du lignage. Son autel est constitué d'un pot dont la base est trouée et recouverte par une pierre plate sur laquelle est posée une masse de pierre et de bâtons, il se trouve généralement au sommet du rocher. Informations recueillies auprès de Météyé, chef de lignage à Gaduwa le 03/10/2017 et Tahapad, fils d'un desservant de MbulumàMasroh le 05/10/2017.

[22] MalaLawdaraoule desservant de l'esprit de l'ancêtre du lignage. Chaque lignage possède un autel autour duquel se rassemble tous les membres du lignage ayant un ancêtre commun. Lawdaraest un esprit de l'ancêtre fondateur du lignage. Tous ses descendants l'honorent et lui rendent un culte collectif une fois l'an. Son autel est symbolisé par une cruche en poterie et est détenuepar le MalaLawdaraqui est un descendant direct de l'ancêtre fondateur. Informations recueillies auprès de Sakoumara desservant de Lawdara, à Masrohle 05/10/2017.

[23] Mahramest un divin guérisseur. Il possède tout d'abord le pouvoir de divination. Les cailloux de quartz et calebasse sont ses instruments. Il intervient dans tous les détails dans la vie des Guemjek. Informations recueillies auprès de Tadasl divin-Guérisseur, à Kwatam, le 08/10/2017.

[24] Klengest le chef-voyant. Il est le surveillant du massif. Il contrôle les sorciers et les esprits maléfiques. Il détecte la présence d'une maladie provoquée par les sorciers. Il peut prédire l'avenir. Il ne procède pas par les méthodes divinatoires, mais il a sa propre vision. Il a un pouvoir mystique. Informations recueillies auprès de Védegwé, voyant, à Gaduwa, le 03/10/2017.

[25] Kerldagamest un desservant de dieu de la porte. Il est le dieu protecteur de la famille. Son autel est constitué de goulot d'un canari fermé par une pierre plate sur laquelle se trouve une masse dont se sert ce dieu pour lapider tout sorcier ou malfaiteur qui tenterait de venir nuire à la famille protégée. Son culte se fait avec les pattes d'animal pendant la maturité du mil.

[26] Entretien avec Kwisseng, 65 ans, chef de massif à Schrem, le 1²r/10/2017.

[27] Manassé, J., « Monographie historique d'un peuple du Mont Mandara : les Guemjek, des origines à nos jours », Mémoire de DIPES II d'Histoire, ENS de Yaoundé I, 2002-2003, pp.34-35

[28] Manassé, J., « Monographie historique d'un peuple du Mont Mandara : les Guemjek, des origines à nos jours », Mémoire de DIPES II d'Histoire, ENS de Yaoundé I, 2002-2003, pp.34-35

[29] Manassé, J., « Monographie historique d'un peuple du Mont Mandara : les Guemjek, des origines à nos 
Gent féminine et pérennisation des valeurs traditionnelles dans les Monts Mandara: cas de la femme podokwo et guemjek à travers le mariage et les tâches quotidiennes

jours », Mémoire de DIPES II d'Histoire, ENS de Yaoundé I, 2002-2003, pp.34-35

[30] Il est par exemple interdit au Bay: le voyage et moins encore de passer la nuit hors de son massif, et si cela arrive, il doit pratiquer un rite pour se réconcilier avec les esprits de la montagne, il ne doit pas partager son plat et ne mange pas en public; il ne doit pas avoir des relations extraconjugales, les activités culinaires s'arrêtent à son domicile à la tombée de la nuit (pas d'art culinaire les nuits), il ne doit jamais transgresser les lois et principes coutumiers.

[31] Entretien avec MaviaWassa, 50ans, Tradi-praticien, à Kassa 1, le 10/09/2017

[32] Entretien avec Bastara Fidel, 65 ans, ancien secrétaire d'Etat civil, à Godigong, le 11/10/2018

[33] Entretien avec Bintou-a-Wadawa, 42 ans, ménagère à Kassa 1, le 11/09/2017

[34] Entretien avec MadamaDouvé, 45 ans, ménagère à Kassa 1, le 11/09/2017

[35] Lembezat, B., Mukulahé, un Montagnard du Nord-Cameroun,Paris, Berger-Levraut, 1952, p.155

[36] Entretien avec Mavia Paul,49 ans, cultivateur à Kassa 1, le 10/09/2017

[37] Entretien avec Mavia Paul,49 ans, cultivateur à Kassa 1, le 10/09/2017

[38] Entretien avec NguéféGojémé, 40 ans, ménagère à Kassa 1 le 11/09/2017

[39] Entretien avec NguéféGojémé, 40 ans, ménagère à Kassa 1 le 11/09/2017

[40] Entretien avec Bintou-a-Wadawa, 42 ans, ménagère à Kassa 1, le 11/09/2017

[41] Entretien avec Bastara Fidel, 65 ans, ancien secrétaired'état civil, à Godigon le 11/10/2018

[42] Hoché Doulouva, «Migrations des populations dites Kirdi du Haut Mandara, des origines à nos jours », Thèse de Doctorat/Ph.D d'histoire non soutenue, Université de Yaoundé I, 2010-2011, pp.29-30

[43] Entretien avec MaviaWassa, 50 ans, Tradi-praticien à Kassa 1, le 10/09/2017

[44] Entretien avec MaviaWassa, 50 ans, Tradi-praticien à Kassa 1, le 10/09/2017

[45] Entretien avec DougdjéMarva, 70 ans, Tradi-praticien, à Kassa 1, le 110/09/2017

[46] Entretien avec DougdjéMarva, 70 ans, Tradi-praticien, à Kassa 1, le 110/09/2017

[47] Cours dispensés par Dr. Odile Chatap dans le cadre de l'Unité de Valeur (U-V. 315) intitulée «Femme et société », Université de Yaoundé I, 2001-2002

[48] Froelich, J.C., «Quelques aspects de la vie socio-économique chez les peuples dits paléo nigritiques » Cahier de l'ISEA, 1968, p.177

[49] Oudjila est un village Podokwo situé à environ $15 \mathrm{~km}$ de Mora. La particularité et la spécificité de sa chefferie traditionnelle résident tout d'abord dans la personnalité même de leur chef Ménégué. Très puissant, il est doté des pouvoirs mystiques sans égal dans tous les massifs podokwo. Dans ses conversations avec ses sujets ou des visiteurs, il ne les regarde pas de face. Il tourne toujours le dos. Dès que vos yeux se croisent, un malheur pourra t'arriver. Enfin, ses innombrables femmes, une quarantaine environ, et une centaine de cases bien construites font l'objet de visites à des touristes.

[50] Entretien avec NguéféGojémé, 4o ans, ménagère à Kassa 1, le 11/O9/2O17.

[51] Les parents peuvent proposer leur fille à un garçon ou à un homme déjà marié, dans une famille qu'ils auraient pointée du doigt, du fait de la bonne moralité, de la serviabilité et pour beaucoup de choses encore.

[52] Ici, dès que l'homme ou un garçon aperçoit la fille, et si la fille remplit physiquement et moralement les conditions souhaitées, il s'informe alors sur ses déplacements (quand est-ce qu'elle sort ? où peut-elle se rendre souvent ?). Si toutes les informations sont réunies, l'homme ou le garçon envoie ses gens pour l'enlever. Dès que l'opération est effectuée, et après quelques jours, il tient informer la famille de la fille pour un accord qui reste dès lors probable.

[53] Dans ce cas, dès le bas âge, l'homme ou le garçon, marque une fille en mettant sur elle une tache de charbon noir. Ce geste n'est pas ludique, mais il est plein de symboles et de portées rituelles. La fille marquée par cette tache de charbon est astreinte d'épouser celui qui l'a marqué, et dans le cas contraire le frère ou l'enfant de celui qui l'a marqué, sinon la mort peut l'emporter dans un bref avenir. Chez les Guemjek, il s'agit d'épouser la femme d'un proche défunt. Selon eux, la richesse, le secret de la famille et la valeur de la femme du proche défunt doivent rester dans la famille.

[54] Il peut arriver que deux amants décident après plusieurs mois d'observations, de fonder ensemble leur foyer à cet effet, ils tiennent informer tous deux leurs familles respectives.

[55] Manassé, J., «Monographie historique d'un peuple du Mont Mandara : les Guemjek, des origines à nos jours» Mémoire de DIPES II, ENS, Yaoundé 1, 2002-2003, p.44.

[56] Entretien avec GoudougwarNgotoko, 60 ans, potière à Gaduwa, le 03/10/2017

[57] Entretien avec TakalDai, 60 ans, sage-femme à Gaduwa, le 03/10/2017 
Gent féminine et pérennisation des valeurs traditionnelles dans les Monts Mandara: cas de la femme podokwo et guemjek à travers le mariage et les tâches quotidiennes

[58] Entretien avec GoudougwarNgotoko, 60 ans, potière à Gaduwa, le 03/10/2017

[59] Le mouillage: ici, les mottes d'argile dure sont pulvérisées, étendues et arrosées sans excès d'eau. L'argile trempée est maintenue toute une nuit ou une journée pour qu'elle soit bien ramollie.

[60] Le pilage: l'argile est pilé à l'aide d'un pilon, ou d'une pierre ovale sur un plateau de bois épais, sur une peau d'animal ou sur un creux aménagé sur une grosse pierre vaste afin d'éliminer les petits cailloux et de rendre l'argile tendre et collante.

[61] Le modelage: il constitue la plus délicate tâche. C'est par le modelage que la potière douée se distingue voire s'illustre. La potière manipule la motte de ses deux mains pour lui imprimer une forme grossièrement cylindrique ou conique suivant la forme générale d'objet à réaliser. L'accès dudit atelier est strictement interdit aux hommes tant que la potière n'a pas fini son travail. L'irruption d'un homme dans l'atelier pourrait avoir un effet néfaste sur les pots et les ustensiles avant ou après la cuisson.

[62] Le façonnage: les techniques diffèrent selon les objets à produire. Lorsque la potière monte les petits vases, elle est assise sur un tabouret en bois contrairement aux grands vases où elle est debout.

[63] La cuisson: après le séchage qui se fait à l'ombre pour éviter les fissures, c'est la phase de cuisson. Elle s'effectue à l'air libre sur une simple aire circulaire largement excavée. Les plus petits sont disposés sur les plus grands. Les vases qui au départ étaient gris, changent de couleur et deviennent rouges ou noirs.

[64] Les jarres pour la conservation d'eau, les grands vases pour préparer le vin traditionnel, les petits vases pour la purification, les grands canaris pour la conservation d'objets précieux, les cuvettes pour fermer les grainiers, et enfin la cruche à forme renflée dotée d'une anse et d'un bec pour les divinités.

\section{Glossaire}

\section{Les mots et expressions podokwo dans le texte}

Batakud-kaya: desservant de l'esprit de la maison

Batatip-Namga/slalawa: faiseur de pluie

Bida: bracelets placés au-dessus du coude et aux aisselles

Doua: jeune fille

Douhoula: jeune garçon

Mundwa/gui-nissa: le mariage

Mtsda: bracelet du poignet

Nissa: la femme

Patcha: rouleaux de perles que les femmes Podokwo attachent aux reins

Taya: chaîne du cou en rouleaux de perles

Tlatawa: la première femme du foyer

Tlewandala: chef des massifs

Tlewandala-mota: chef des cultes, grand prêtre

\section{Les mots et expressions guemjek dans le Texte}

Bay: le chef

Bay-Gma: le chef des massifs

Duwa: lespotsàusage domestique

Fétékoukerngai:l'éternel pour désigner le chef

Gwala-bay:la classe détentrice du pouvoir

Hotokom: le sommet pour désigner le chef

Kerldagam: le desservant des dieux de la porte

Klen: voyant,

Kur-hembet: pierre de tornade

Kur-kula: pierre de sécheresse 
Gent féminine et pérennisation des valeurs traditionnelles dans les Monts Mandara: cas de la femme podokwo et guemjek à travers le mariage et les tâches quotidiennes

Kur-yam: pierre de pluie

Lawdara: esprit de l'ancêtre d'un lignage

Mahram: le divin guérisseur

Mbulum: esprit des lieux

Mala-yam: faiseur de pluie

\section{AUTHOR BIOGRAPHY}

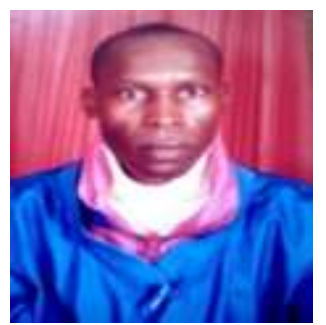

Tougbouné est doctorant en phase finale de sa Thèse de Doctorat/ Ph.D d'Histoire à l'Université de Maroua. Son domaine de recherches est l'Histoire sociale et politique. Il a été Chef de Service de la Scolarité et de Diplômes à l'École Normale Supérieure de l'Université de Maroua. Tougbouné est présentement chargé de la Bibliothèque de Recherches (Sciences de l'Homme et de la Société) logée à l'Ecole Normale Supérieure de l'Université de Maroua.

Citation: Tougbouné. "Gent féminine et pérennisation des valeurs traditionnelles dans les Monts Mandara: cas de la femme Podokwo et Guemjekà travers le mariage et les tâches quotidiennes". International Journal of Humanities Social Sciences and Education (IJHSSE), vol.6, no.10, 2019, pp. 26-37. doi: http://dx.doi.org/10. 20431/2349-0381.0610003.

Copyright:@ 2019 Authors. This is an open-access article distributed under the terms of the Creative Commons Attribution License, which permits unrestricted use, distribution, and reproduction in any medium, provided the original author and source are credited. 